\title{
Vertex Tracking at a Future Linear Collider
}

\author{
M. Battaglia \\ Santa Cruz Institute of Particle Physics, University of California at Santa Cruz, Santa Cruz CA 95064, USA \\ Lawrence Berkeley National Laboratory, Berkeley CA 94720, USA \\ CERN, Geneva CH-1211 Switzerland
}

\begin{abstract}
The anticipated physics program at an high energy $e^{+} e^{-}$linear collider places special emphasis on the accuracy in extrapolating charged particle tracks to their production vertex to tag heavy quarks and leptons. This paper reviews physics motivations and performance requirements, sensor R\&D directions and current results of the studies for a vertex tracker at a future linear collider.
\end{abstract}

Keywords: Pixel detectors, linear colliders

\section{Introduction}

A high energy $e^{+} e^{-}$linear collider has emerged as possibly the most practical and realistic way towards collisions of elementary particles at constituent energies matching those of the LHC with high luminosity. The ILC project is based on the use of superconducting RF cavities providing gradients of $\simeq 30 \mathrm{MV} / \mathrm{m}$ to produce collisions at centre-of-mass energies $\sqrt{\mathrm{s}}=$ $0.25-1 \mathrm{TeV}[1]$. In order to achieve multi-TeV $e^{+} e^{-}$ collisions, the CLIC project develops a new acceleration scheme where a low-energy, high-current drive beam is used to accelerate the main beam through highfrequency transfer structures, which have achieved gradients in excess of $100 \mathrm{MV} / \mathrm{m}$ [2].

Heavy flavours represent an essential signature of the anticipated physics of interest. The study of the Higgs sector of the Standard Model, of TeV-scale new physics and the search for new phenomena at very high mass scale through electro-weak precision observables, all depend on the identification and decay reconstruction of $t, b$ and $c$ quarks and of $\tau$ leptons [3]. This physics program requires a vertex tracker able to extrapolate the particle tracks back to their production vertex with high accuracy over a broad momentum range. The linear collider requirements have motivated a vigorous and diversified R\&D program which has seen monolithic pixels of various technologies emerging as a mature and wellperforming option for vertex tracking applications.

Email address: MBattaglia@lbl.gov (M. Battaglia)

\section{Tracking Accuracy, Flavour Tagging and Experi- mental Conditions}

The standard figure of merit for tracking accuracy is the resolution on the impact parameter, $\sigma_{I P}$, defined as the distance of closest approach of the particle track to the colliding beam position. This can be parametrised as:

$$
\sigma_{I P}=a \oplus \frac{b}{p \sin ^{k} \theta}
$$

where $\theta$ is the track polar angle and $k=3 / 2$ for the $R-\Phi$ and $5 / 2$ for the $z$ projection. The target parameters for a $0.25-0.5 \mathrm{TeV}$ collider are $a=5 \mu \mathrm{m}$ and $b=$ $10 \mu \mathrm{m} \mathrm{GeV}^{-1}$.

The identification of hadronic jets originating from heavy quarks is best achieved by a topological reconstruction of the displaced secondary and tertiary vertex structure and the kinematics associated to $B$ hadron decays. The ability to reconstruct the sequence of primary, secondary and tertiary vertices depends on the impact parameter resolution. Jet flavour tagging for the linear collider extends the strategy successfully adopted in SLD, to date the collider experiments with the best track extrapolation accuracy [4]. The ZVTOP algorithm [5], originally developed for physics at SLC and now adapted for use at a linear collider, demonstrated high $b$-tagging efficiency on fully simulated and reconstructed events [6]. The impact of a change of the $a$ and $b$ parameters in (1) on the physics performance have been studied on detailed simulation and reconstruction. In $500 \mathrm{GeV}$ energy $b$-jets, doubling the values of $a$ and $b$ 
from $3 \mu \mathrm{m}$ and $18 \mu \mathrm{m} \mathrm{GeV}-1$ to $6 \mu \mathrm{m}$ and $36 \mu \mathrm{m} \mathrm{GeV}-1$, respectively, results in a $15 \%$ decrease of both the number of vertices reconstructed in the $B$ decay chain and the fraction of particle tracks correctly assigned to their vertex of origin. The efficiency for the identification of $b$ jets at a constant purity of 0.90 , in a sample where light, $c$ and $b$ flavours are uniformly represented, drops from 0.75 for $a=5 \mu \mathrm{m}$ and $b=10 \mu \mathrm{m} \mathrm{GeV}^{-1}$ to 0.25 for $a=12 \mu \mathrm{m}$ and $b=70 \mu \mathrm{m} \mathrm{GeV}{ }^{-1}$. That for tagging $c$-jets at a purity of 0.70 drops from 0.50 for $a=$ $5 \mu \mathrm{m}$ and $b=10 \mu \mathrm{m} \mathrm{GeV}^{-1}$ to 0.29 for $a=11 \mu \mathrm{m}$ and $b=15 \mu \mathrm{m} \mathrm{GeV}^{-1}$ [7]. Propagating these effects to the statistical accuracy of physics measurements, such as Higgs decay branching fractions, generally shows that a degradation of a factor of two on the $a$ or $b$ terms of (1) corresponds to a $20-30 \%$ equivalent luminosity loss at $0.5 \mathrm{TeV}[8,9]$.

Multiple $t$ and $b$ quarks are expected to be a distinctive feature in several processes in multi- $\mathrm{TeV} e^{+} e^{-}$collisions. The signal cross sections are typically of $O(1 \mathrm{fb})$ with signal-to-background ratios of $10^{-2}-10^{-5}$ and two to six heavy flavour jets. The energy of $b$ jets ranges from $50 \mathrm{GeV}$ up to the full beam energy of $\sim 1.5 \mathrm{TeV}$. In the benchmark process $e^{+} e^{-} \rightarrow H^{0} A^{0} \rightarrow b \bar{b} b \bar{b}$, with $M_{A}$ around $1 \mathrm{TeV}$ at $\sqrt{s}=3 \mathrm{TeV}$, jets have $B$ hadrons flying on average $27 \mathrm{~mm}$ and approximately one third of them decays at a radius larger than $30 \mathrm{~mm}$. In addition, forward $b$-tagging is crucial to study some specific SM processes, which may only be accessible to a multi-TeV $e^{+} e^{-}$collider, such as the triple Higgs coupling, through $e^{+} e^{-} \rightarrow v_{e} \bar{v}_{e} h^{0} h^{0}$, and the fermionic coupling of an intermediate-mass Higgs boson, through $e^{+} e^{-} \rightarrow v_{e} \bar{v}_{e} h^{0} \rightarrow v_{e} \bar{v}_{e} b \bar{b}$. In this case, the $B$ hadron energy is in the range $25<E<300 \mathrm{GeV}$, due to the large energy taken by the neutrinos.

The track extrapolation requirements outlined above can be met with a vertex tracker which has thin layers, first measurement close to the beam interaction point and excellent single point resolution. Considering a traditional barrel geometry with two layers located at radii $R_{\text {in }}$ and $R_{\text {out }}$, the asymptotic impact parameter resolution $a$ is given by:

$$
a=\sqrt{(n+1)^{2}+n^{2}} \sigma_{\text {point }}
$$

where $n=\frac{R_{i n}}{R_{\text {out }}-R_{\text {in }}}$ and $\sigma_{\text {point }}$ is the single point resolution. With $R_{\text {in }}=15-30 \mathrm{~mm}$ and a lever arm $R_{\text {out }}-R_{\text {in }}$ of $30-40 \mathrm{~mm}$, the requirement $a \leq 5 \mu \mathrm{m}$ implies a point resolution $\sigma_{\text {point }} \leq 3.5 \mu \mathrm{m}$. The requirement on the pixel pitch, $P$, comes from the point resolution $\left(\sigma_{\text {point }} \leq P / \sqrt{12}\right.$ for binary readout and $\propto P /(S / N)$ for analog readout with charge interpolation) but also from the two-track separation $(\propto P)$ and from the occupancy $\left(\propto P^{2}\right)$. Single point resolutions equal to, or better than, that required for the linear collider have been obtained in several monolithic pixel technologies, including CMOS MAPS [10], DEPFET [11] and SOI [12], for pixel pitches in the range $10-30 \mu \mathrm{m}$ and $\mathrm{S} / \mathrm{N}$ values of 20 to 130. In particular, CMOS sensors with binary output have demonstrated a detection efficiency $>99 \%$, fake rate $<10^{-4}$ and point resolution of $3.5 \mu \mathrm{m}$, below the $P / \sqrt{12}$ limit of their pixel pitch of $18.4 \mu \mathrm{m}[10,13$.

The multiple scattering term, $b$, is given by:

$$
b=\sum_{i} R_{i}^{2} \theta_{0, i}^{2}
$$

where the index $i$ runs over the material surfaces traversed by the particles and $\theta_{0}$ is the multiple scattering angle for normal incidence tracks. It is desirable to install the first layer as close as possible to the interaction point to minimise the multiple scattering effect and optimise the asymptotic resolution. In practice this radius is set by the density of low momentum pair electrons and positrons.

Pairs are produced by beam particles scattering on real and virtual photons created in the intense beambeam electromagnetic interactions. The intrinsic $p_{t}$ of most of these electrons is small but they get deflected in the electric field of the incoming beam and can reach the detector. Incoherent pairs, spiralling in the solenoidal magnetic field of the detector, form an envelope, whose radius at a given position $z$ along the beam axis defines a lower bound on the position of the innermost detector layer. This bound scales approximately as:

$$
R \sim \sqrt{\frac{N}{10^{10}} \frac{1}{\sigma_{z}} \frac{1}{B} z .}
$$

$N$ is the number of particles in a bunch $\left(=2 \times 10^{10}\right.$ for ILC and $3.7 \times 10^{9}$ for CLIC), $\sigma_{z}$ is the bunch length (= $300 \mu \mathrm{m}$ for ILC and $40 \mu \mathrm{m}$ for CLIC) and $B$ the solenoidal field $(=3-5 \mathrm{~T}$ depending on the detector concept design) [14, 15]. Outside the deflected pair envelope there is a residual pair population, due to large $p_{t}$ electrons and to particles deflected at large angles or back-scattered from the inner face of the low angle calorimeter, which is located downstream from the interaction point. The ILC has a predicted hit density from incoherent pairs of 4.4 hits $\mathrm{mm}^{-2} \mathrm{BX}^{-1}$ at a radius of $16 \mathrm{~mm}$ and $\sqrt{s}=0.5 \mathrm{TeV}$, which increases by approximately a factor of two by doubling the beam energy or changing the beam parameters to achieve a constant luminosity. At $\sqrt{s}=3 \mathrm{TeV}, \mathrm{CLIC}$ has $\simeq 2.2$ hits $\mathrm{mm}^{-2}$ 
$\mathrm{BX}^{-1}$ at a radius of $31 \mathrm{~mm}[16]$. The factor two increase in the radius of the vertex tracker innermost layer at CLIC compared to the ILC results in a change of the multiple scattering term from $10 \mu \mathrm{m} \mathrm{GeV}^{-1}$ to $21 \mu \mathrm{m}$ $\mathrm{GeV}^{-1}$. Pairs are also responsible for an ionising dose of $\sim 100 \mathrm{krad} /$ year to be added to a non-ionising dose corresponding to $\simeq 7 \times 10^{10} \mathrm{n}_{e q} \mathrm{~cm}^{-2}$ year $^{-1}$ from pairs, which is larger than that due to neutrons, estimated to $\simeq$ $10^{10}$ neutrons $\mathrm{cm}^{-2}$ year $^{-1}$ at $0.5 \mathrm{TeV}$ [17]. These figures are expected to be comparable, $\sim 100 \mathrm{krad} /$ year and $5 \times 10^{10} n \mathrm{~cm}^{-2} \mathrm{year}^{-1}$, for CLIC at $3 \mathrm{TeV}$ [18].

Experience in tracking and vertexing with prototype monolithic pixel sensors has already been obtained by several groups. In particular, the EUDET project and the DEPFET group have extensive experience with tracking beam particles. The EUDET telescope consists of two arms each equipped with three layers of $50 \mu \mathrm{m}$ thin CMOS MAPS chips with in-pixel correlated double sampling, column parallel readout with discriminator and zero suppression logic at the end of the column. It provides a track extrapolation accuracy of 1-2 $\mu \mathrm{m}$ on the detector under test. Using a readout time of $100 \mu \mathrm{s}$ it can operate with large track density, up to $10^{6}$ particles $\mathrm{cm}^{-2} \mathrm{~s}^{-1}$ [19]. The T966 beam test experiment also operated a beam telescope made of $50 \mu \mathrm{m}$-thick CMOS pixel sensors [20]. Four sensors were arranged with a $15 \mathrm{~mm}$ spacing which is close to that proposed for the ladders of a Vertex Tracker at the linear collider. It studied the tracking extrapolation accuracy for $1.5 \mathrm{GeV}$ electrons at the LBNL Advanced Light Source (ALS) and $120 \mathrm{GeV}$ protons at the Fermilab Test Beam Facility. With a point resolution of $2.3 \mu \mathrm{m}$, the accuracy for extrapolating the reconstructed particle track by $15 \mathrm{~mm}$ upstream was measured to be $(8.5 \pm 0.4) \mu \mathrm{m}$ and (4.2 \pm 0.3$) \mu \mathrm{m}$ at 1.5 and $120 \mathrm{GeV}$, respectively, which matches the linear collider requirements. In addition, the vertexing accuracy was studied for $p$ interactions in a thin $\mathrm{Cu}$ target located $32 \mathrm{~mm}$ upstream from the first sensor, corresponding to the distance between the first vertex layer and the interaction point foreseen at CLIC. The longitudinal vertex position resolution of $260 \mu \mathrm{m}$ for an average track multiplicity of 2.74 closely matches that expected for CLIC of $220 \mu \mathrm{m}$ for reconstructed secondary decays vertices of $B$ hadrons having an average track multiplicity of 3.02 .

\section{Low-mass ladders for the Vertex Tracker}

Despite the large design collision energies, charged particles are typically produced with moderate energies, due to the large jet multiplicity or missing energy. $b$ jets are discriminated from $c$ jets based on the number and invariant mass of the secondary particles. This requires most, if not all, of the $b$ charged decay products to be identified. Excellent track extrapolation at low momenta is therefore essential. At $0.5 \mathrm{TeV}$ the $c$ tagging efficiency, at constant purity, in the study of $h^{0} \rightarrow c \bar{c}$, drops by $25 \%$ when changing the ladder thickness from $0.1 \% X_{0}$ to $0.3 \% X_{0}$ [21]. The multiple scattering term $b$ plays even a more crucial role than the asymptotic resolution $a$, in particular for processes which are forward peaked. The fraction of charged $B$ decay products identified as secondaries based on their impact parameter significance, $I P / \sigma_{I P}$, in $e^{+} e^{-} \rightarrow v_{e} \bar{v}_{e} h^{0} \rightarrow$ $v_{e} \bar{v}_{e} b \bar{b}$ events at $\sqrt{s}=3 \mathrm{TeV}$, drops from 0.85 for $b=$ $15 \mu \mathrm{m} \mathrm{GeV}^{-1}$ to 0.74 for $35 \mu \mathrm{m} \mathrm{GeV}^{-1}$. For comparison, it changes by just $2 \%$ for $1.5<a<3.5 \mu \mathrm{m}$.

Chips of various technologies have been successfully thinned to $\leq 100 \mu \mathrm{m}$. Assuming that the sensors are mounted on an $100 \mu$ m-thick carbon fiber composite (CFC) ladder, there is little benefit for the multiple scattering term from pushing their thickness below $\sim 50 \mu \mathrm{m}$. It has been shown that CMOS chips can be thinned to $50 \mu \mathrm{m}$ with high yields and no loss in performance [22]. For DEPFET sensors an interesting method of selective thinning of the handle wafer in the sensitive area while retaining a support frame for mechanical stability has been developed on large area prototypes and should be soon tested on sensor chips [23]. Several designs of low material ladders have been proposed, based on specific sensor technologies. The ladders of the STAR HFT are probably the most advanced prototypes built so far. Based on CMOS MAPS thinned to $50 \mu \mathrm{m}$, the ladder has a total material budget of $0.37 \% X_{0}$, needed for the study of low- $p_{t} D$ meson production in heavy ion collisions at RHIC [24]. The chosen geometry, with two barrel layers at 25 and $80 \mathrm{~mm}$ radius, will obtain a multiple scattering $b$ term of $19 \mu \mathrm{m} \mathrm{GeV}{ }^{-1}$. In the DEPFETbased ladder proposed for Belle-II the material budget decreases to $0.19 \% X_{0}$ which becomes $0.17 \% X_{0}$ in the conceptual design of a FPCCD-based double-layer ladder envisaged for the ILC. It is interesting to observe that in both the STAR HFT and the Belle-II ladders the Si chip accounts for less than half of the ladder material budget. In particular, the kapton cable with Al traces installed below the sensors to route signals, power and clocks accounts in the STAR design for $20 \%$ of the total material budget of the ladder. In order to progress towards thinner modules, sensor stitching, with clock and signals routed on metal lines in the chips, appears a promising path.

Different arrangements of detectors in a multi-layered barrel geometry are being evaluated. The first is a traditional vertex tracker consisting of five equally-spaced 
layers with thin Si chips mounted on a light-weight carbon composite ladder support. Measurements carried out at LBNL with an optical survey machine on a freestanding $1.9 \times 1.9 \mathrm{~cm}^{2}$ CMOS MAPS chip thinned to $40 \mu \mathrm{m}$ show a warping which is primarily cylindrical. The measured peak-to-peak amplitude is $1.7 \mathrm{~mm}$, which results in a significant stress when the chip is flattened on its carrier [25]. The support structure must counter this deformation and ensure the planarity of the sensors on the ladder. An interesting solution is the use of a double-sided ladder made of a carbon foam layer with sensors mounted at both sides, in a sandwich-like structure. This design places the thin pixel chips backto-back on the same carrier minimising ladder bending, thanks to the symmetric layout, and reducing the material budget per active layer [25, 26]. It also provides hit position correlation on the closely-spaced detector planes, which may offer further advantages for redundancy and rejection of low-energy electrons from the pair background. The PLUME R\&D program foresees the development of light-weight double-layer ladder prototypes, based on CMOS sensors, with the aim to achieve a material budget of $0.2 \% X_{0}$ [27].

The LHC detector experience shows that cooling and services dominate the total material budget. Passive cooling appears necessary to maintain the ladder material within few per-mil of a radiation length. A simple air flow is adopted by the STAR HFT. Tests carried out at LBNL have shown that airflow at $10 \mathrm{~m} \mathrm{~s}^{-1}$ can remove up to $\simeq 200 \mathrm{~mW} \mathrm{~cm}^{-2}$ with an acceptable temperature rise above ambient [24, 25]. Results reported by the SiLC collaboration confirm this figure [28]. This places stringent constraints on the chip power dissipation which must be reconciled with the requested data processing and readout speed. The MIMOSA-26 CMOS chip, realised in the framework of the EUDETFP6 project and which represents a prototype for the sensor for the STAR HFT, has an average power dissipation of $280 \mathrm{~mW} \mathrm{~cm}^{-2}$ [13]. More advanced thermal management approaches, such as micro-channel cooling, are becoming more realistic following recent developments in the IC industry and are presently being evaluated [29].

\section{Sensor Technologies and Architectures for the Vertex Tracker}

One of the most demanding requirements for the sensor architecture at a linear collider is the mitigation of the anticipated occupancy due to incoherent pairs, within the basic specifications of $\leq 3.5 \mu \mathrm{m}$ single point resolution, $\simeq 50 \mu \mathrm{m}$ thickness, power dissipation $\leq 200 \mathrm{~mW} \mathrm{~cm}^{-2}$ (assuming airflow cooling). This can be achieved either by an architecture based on fast continuous readout or by in-situ charge storage with high space (or space-time) granularity and read-out at the end of the bunch train. At the ILC, which has 2820 bunches/train with a $337 \mathrm{~ns}$ spacing and $5 \mathrm{~Hz}$ repetition rate, an occupancy low enough not to affect the pattern recognition should be achievable on the innermost layer by a readout within 25-50 $\mu$ s. An example of continuous readout architecture is implemented on CMOS MAPS with double-sided, column-parallel binary read-out using a rolling shutter, correlated double sampling and zero suppression [13]. DEPFET pixels are read-out 20 times during the train, with data stored on the chip periphery [30]. Alternatively, time-stamping can be performed to divide the bunch train into several time-buckets. Concepts currently under R\&D are the Chronopixels [31] and the ISIS pixels with in-situ 20cell charge storage [32, 33]. Finally, small enough pixels can achieve a low occupancy even integrating over a full bunch train. The FPCCD concept is based on an extreme space granularity, where $5 \times 5 \mu \mathrm{m}^{2} \mathrm{CCD}$ pixels ensure low occupancy and, possibly, background hit rejection through cluster shape analysis [34].

Continuous readout schemes must ensure immunity from pick-up during the bunch train and are challenging for background rates significantly higher than the current estimates for the ILC. In-pixel time stamping, as pursued by the Chronopixel design, has to achieve noise reduction and high bandwidth within an acceptable power budget. The design targets a $45 \mathrm{~nm}$ CMOS process with deep implants to avoid competitive charge collection, still untested for pixel detectors. At an $\mathrm{X}$-band multi-TeV collider, such as CLIC, both the bunch spacing $(0.5 \mathrm{~ns})$ and the train length (150 ns) are too short to achieve a reduction of the integrated background by fast readout. Instead, high space-time granularity, such as time-stamping to $15-30 \mathrm{~ns}$, must be considered, given the expected background rate of $\sim 5$ hits $\mathrm{mm}^{-2}$ per bunch train. Integrating fast time stamping in a thin sensor, with small pixel cells and limited power consumption requires significant R\&D, which can profit of features made available by emerging semiconductor technologies.

The R\&D driven by the ILC specifications has made significant progress in the last decade towards sensors which are now ready for applications in real experiments (STAR, CBM, Belle-II) with specifications which are in many areas close to those of a linear collider. At STAR, where there are $\simeq 2$ particles $\mathrm{mm}^{-2}$ in the $200 \mu \mathrm{s}$ integration time, sensors with binary output and on-chip zero suppression are foreseen for installation in 2013. 
The expected ionising does is $\sim 100 \mathrm{krad} /$ year. At BelleII the hit density is expected to be $\simeq 8$ hits $\mathrm{mm}^{-2}$ in the $20 \mu$ s integration time of a DEPFET detector, which makes it an even bigger challenge compared to a linear collider. The ionising dose should exceed $1 \mathrm{Mrad} /$ year. The construction and operation of these detectors will also provide a valuable experience with those aspects which have been studied less so far, such as yields, system issues and operation reliability.

Beyond particle physics, new applications in imaging and X-ray detection have been made possible by the linear collider R\&D. Imaging in transmission electron microscopy (TEM) with CMOS sensors is under fast development and profits from the availability of thin sensors with thin sensitive volume to minimise low energy electron scattering and thus the point spread function [35, 36, 37]. Given that a very intense bright field image could deposit order of $10 \mathrm{rad} \mathrm{s}^{-1}$ pixel $^{-1}$, a target radiation tolerance of $\geq 1 \mathrm{Mrad}$ is a valid requirement. The development of sensors for TEM applications has contributed useful results in terms of design and tests of radiation-tolerant pixel cells, able to operate after doses of several Mrad [37]. The integration of CMOS sensors into photosensitive systems for fluorescent and bioluminescent high-speed imaging in biology has already provided a characterisation of a first prototype with different fluorescent dyes [38]. Low noise and high energy resolution make DEPFET sensors ideal for X-ray spectroscopy at high rates. Applications both at the European XFEL [39] and for the focal plane of a planetary observation satellite experiment [40] are presently being developed.

Despite the successes in the development of a new generation of pixel sensors for application at a linear collider, there are still several areas where more sensor R\&D is needed. Fast charge collection and signals larger than the $\leq 10^{3}$ electrons generated in the thin epitaxial layer of conventional bulk CMOS processes are highly desirable to ensure radiation tolerant devices and the possibility of fast time stamping. The integration of not only signal sensing but also advanced data processing in pixel, or at least on chip, will also profit from the introduction of new technologies. The port of MAPS to commercially available processes with an high resistivity epitaxial layer or substrate is actively pursued. These offer higher charge yield, faster charge collection dominated by drift and improved radiation tolerance compared to conventional bulk CMOS technology. MIMOSA sensors with high-resistivity epilayer have been successfully tested and will be used in the STAR HFT [10]. CMOS pixel sensors with an high-resistivity sensitive volume are being developed by the LEPIX collaboration centred at CERN in a custom $90 \mathrm{~nm}$ process. The first structures are presently being evaluated. The Silicon-On-Insulator process with an high-resistivity handle wafer offers further appealing opportunities by removing limitations of bulk CMOS processes. After the pioneering effort in a $3 \mu \mathrm{m}$ CMOS process of [41], a commercial $0.2 \mu \mathrm{m}$ SOI process has been made available since a few years by a collaboration of KEK with OKI Semiconductors, Japan, [42]. The high resistivity sensitive volume ensures large signals with no interconnections, low collection electrode capacitance and a low-power, potentially radiation tolerant device [43, 44]. The R\&D has successfully solved the transistor back-gating problem. Prototype SOI pixels recently tested with a $200 \mathrm{GeV} \pi^{-}$beam at CERN have been successfully operated with a depletion voltage up to $70 \mathrm{~V}$, giving a preliminary result of $(1.2 \pm 0.05) \mu \mathrm{m}$ single point resolution and particle detection efficiency above $98 \%$ [12]. Similar features, but allowing also the integration of heterogeneous CMOS technologies can be obtained using 3D multi-tier vertical integration of thin Si chips. Several pilot R\&D programs have recently been launched to explore the use of $3 \mathrm{D}$ interconnectivity for pixel sensors and results are expected soon [44, 45].

\section{Conclusions}

Physics requirements at a future linear collider have motivated a significant R\&D effort on monolithic pixel sensors to achieve small pixel cells with integrated charge sensing and data processing, thin sensors with low power consumption and high space-time granularity. Current studies show that moving from a sub-TeV to a multi-TeV collider implies new requirements especially on forward vertexing capabilities and detector fast time stamping, which need to be addressed by a dedicated R\&D program. The LHC data will likely indicate the energy range of interest for a linear collider in the next few years. Despite the long time scale of these programs, technologies developed in the ILC-motivated R\&D have already demonstrated a significant impact on other particle physics experiments as well as imaging and spectroscopy applications in other fields of science from electron microscopy to biology and astronomy.

\section{Acknowledgement}

I am grateful to the organisers of the PIXEL 2010 workshop for their invitation and an enjoyable conference and to L. Andricek, F. Bogert, J. Brau, D. Contarato, R. De Masi, P. Denes, P. Giubilato, L. Greiner, 
A. Sailer, D.B. Shuman, W. Snoeys and Y. Sugimoto for their contributions.

\section{References}

[1] J. Brau et al., SLAC-R-857 (2007).

[2] R. W. Assmann et al., CERN 2000-008.

[3] M. Battaglia, to appear on Nucl. Phys. (2010) arXiv:1009.4184 [physics.hep-ex]].

[4] T. Abe [SLD Collaboration], Nucl. Instr. Meth. A 447 (2000) 90 arXiv:hep-ex/9909048].

[5] D. J. Jackson, Nucl. Instr. Meth. A 388 (1997) 247.

[6] D. Bailey et al. [LCFI Collaboration], Nucl. Instr. Meth. A 610 (2009) 573 [arXiv:0908.3019 [physics.ins-det]].

[7] R. Hawkings, LC-PHSM-2000-021.

[8] T. Kuhl and K. Desch, LC-PHSM-2007-001.

[9] G.B. Yu et al., J. Korean Phys. Soc. 50 (2007) 10.

[10] M. Winter, Nucl. Instr. Meth. A 623 (2010) 192.

[11] P. Kvasnicka [DEPFET Collaboration], to appear on Nucl. Instr. Meth. A (2010) doi:10.1016/j.nima.2010.06.170.

[12] P. Giubilato et al., in this volume.

[13] R. De Masi et al., to appear on Nucl. Instr. Meth. A (2010) doi:10.1016/j.nima.2010.06.339.

[14] M. Battaglia et al., Proc. of the $2^{\text {nd }}$ Workshop on Backgrounds at the Machine Detector Interface, T.E. Browder and S.K. Sahu (Editors), World Scientific, Singapore 1998, 144.

[15] M. Battaglia, Nucl. Instr. Meth. A 530 (2004) 33 |arXiv:physics/0312039].

[16] A. Sailer, CERN LCD-Note-2010-007.

[17] A. Besson et al., Nucl. Instr. Meth. A 568 (2006) 233.

[18] M. Battaglia, A. De Roeck, J. R. Ellis and D. Schulte (editors), CERN-2004-005, |arXiv:hep-ph/0412251].

[19] C. Hu-Guo et al., Nucl. Instr. Meth. A 623 (2010) 480.

[20] M. Battaglia et al., Nucl. Instr. Meth. A 593 (2008) 292 [arXiv:0805.1504 [physics.ins-det]].

[21] P. Luzniak, arXiv:0902.0073 2 [physics.ins-det].

[22] M. Battaglia et al., Nucl. Instr. Meth. A 579 (2007) 675 arXiv:physics/0611081].

[23] P. Fischer et al., Nucl. Instr. Meth. A 582 (2007) 843.

[24] L. Greiner et al., in this volume.

[25] M. Battaglia et al., Report LBNL-63495 (2007).

[26] H. Stoeck et al., The ILD Letter of Intent, 2009.

[27] A. Nomerotski et al., in this volume.

[28] A. Savoy Navarro et al., EUDET Memo 2007-52.

[29] F. Bosi et al., in this volume.

[30] J.J. Velthuis et al., Nucl. Instr. Meth. A 579 (2007) 685.

[31] J. Brau et al., Nucl. Instr. Meth. A 579 (2007) 567.

[32] J.J. Velthuis et al., Nucl. Instr. Meth. A 599 (2009) 161.

[33] C. Damerell et al., Nucl. Instr. Meth. A 624 (2010) 465.

[34] Y. Takubo et al., Nucl. Instr. Meth. A 623 (2010) 489.

[35] A.-C. Milazzo et al, Ultramicroscopy 104 (2005) 152.

[36] G. Deptuch et al., Ultramicroscopy 107 (2007) 674.

[37] M. Battaglia et al., Nucl. Instr. Meth. A 622, (2010) 669.

[38] R. Barbier et al., Nucl. Instr. Meth. A 610, (2009) 54.

[39] M. Porro et al., Nucl. Instr. Meth. A 624 (2010) 509.

[40] J. Treis et al., Nucl. Instr. Meth. A 624 (2010) 540.

[41] J. Marczewski et al., Nucl. Instr. Meth. A 549 (2005) 112.

[42] Y. Arai et al., to appear on Nucl. Instr. Meth. A (2010) doi:10.1016/j.nima.2010.04.081

[43] M. Battaglia et al., Nucl. Instr. Meth. A 583 (2007) 526 arXiv:0709.4218 [physics.ins-det]].

[44] Y. Arai et al., Nucl. Instr. Meth. A 623 (2010) 197.

[45] R. Yarema et al., Nucl. Instr. Meth. A 617 (2010) 375. 